Full length article

\title{
MODELING AND ANALYSIS OF MAGNETIZED CHEMICALLY REACTIVE FLUID FLOW PAST OVER POROUS STRETCHED SHEET
}

\author{
F. Haq ${ }^{*}$, M. U. Rahman' and S. Hussain' \\ 1 Department of Mathematical Sciences, Karakoram International University, Gilgit, Pakistan
}

\begin{abstract}
The aim of this article to inspect the effect of nonlinear thermal radiation, heat joule, viscous dissipation and magnetic field on viscoelastic second grade fluid. Flow is generated due to stretching of sheet. Flow features are studied considering hydrodynamic boundary conditions. Chemical reaction on the surface is further accounted. The flow governing nonlinear partial system of differential equations is obtained incorporating boundary layer assumptions. The dimensional model is made dimensionless by taking suitable transformations and then tackled via HAM for convergent series solution. Effects of flow controlling parameters on velocity, concentration, temperature, local skin friction coefficient, Sherwood number and Nusselt numbers are discussed by plotting graphs. Main observations are listed at the end.
\end{abstract}

KEYWORDS: Fluid flow, porous media, HAM

*Corresponding author (email: fazal.haq@kiu.edu.pk)

\section{INTRODUCTION}

Magnetohydrodynamics(MHD) is a part of science which describes the relation between the magnetic fields and moving electrically conducting fluid. Hydromantic phenomenon becomes more important due to its practical applications in engineering, physics chemistry and industries, like MHD throttles, automatic fuel level indicator, nuclear reactor, magnetometer, electronic motors and transformer. The origin of hydromantic was first time introduced by Alfven[1]. Later on several researchers investigated MHD effects on the flow over different geometries [2-7]. Nadeem et al. [8] studied MHD liquid flow over shrinking sheet. Analytical and numerical solution of MHD boundary layer flow problem over an unsteady stretching sheet is reported by Sheikholeslami [9].

The flow field study near stretching sheet in boundary layer flow is an important process in fluid dynamics and engineering. The heat transfer process occurring in different engineering processes such as crystal growth, plastic sheets, glass fiber, polymer processing and metallurgy [10]. Ghosh et al. [11] applied Laplace transformation to study the behavior of flow of MHD viscoelastic incompressible fluid having small particles and moving between two parallel plates of infinite length. To investigate heat transfer in MHD viscoelastic fluid flow in presence of thermal radiation over a semi-infinite, non-isothermal stretching impermeable sheet with internal heat generation/ absorption, Datti et al. [12] applied fourth-order RK-4 (Runge-Kutta-4) method. 
Analysis of Dufour effects on mass and heat transmission in micro polar liquid flow over an isothermal sphere Keller-box implicit technique is applied by Beg et al.[13]. Kamel et al.[14] used Laplace transformation technique to study MHD flow of vertical permeable sheet of infinite length. Chen et al. [15] used a central-difference scheme to deliberate the influence of involved physical parameters in governing equations power-law stretched sheet of non-Newtonian power-law fluids past with surface heat flux.

Invent of modern high speed computers and development of new methods/techniques has a significant role in solving highly nonlinear problems. To solve nonlinear problems most of the researchers used analytical methods. These methods are reliable and have high convergence then other methods [16]. Homotopy Analysis Method (HAM) is one of reliable and efficient technique to get the analytical solution of nonlinear differential equations. HAM was introduced by Liao, as an analytical method for finding the solution of nonlinear problems [17]. Khan et al.[18] studied heat transfer in Magnetohydrodynamic Sisko fluid through a porous medium. Dufour and Soret's effects over a vertical stretching sheet on mixed convection of a viscoelastic fluid flow is studied by Hayat et al.[19] using HAM. To study the behavior of unsteady flow in case of heat transfer over stretching sheet Rashdi et al.[20] used HAM. Maxwell fluids heaving mixed convection effects in a boundary layer over a vertical stretching surface is studied by Abbas et al. [21] via HAM.

Our main concern here is to scrutinize the magnetized flow of viscoelastic second grade fluid over stretched sheet. Thermal radiation, viscous dissipation and heat source are considered in energy relation. Concentration communication is modeled in view of chemical reaction. The impact of sundry variable on heat transfer, mass transfer, fluid velocity, temperature and concentration are analyzed through plots.

\section{PROBLEM FORMULATION}

In this study we consider 2-D boundary-layer flow of visco-elastic fluid over a stretching and electrically conducting sheet. The electrically conducting fluid through applied magnetic field $B_{0}$ via thermal radiation, heat transfer characteristics is explored. Furthermore Joule heating and dissipation are also carried. Let us assume that $u(x)=b x$ is strains velocity in flow (see Fig. 1) direction and $T$ and $C$ are temperature and concentration of fluid. The governing equations in view of above assumptions are:

$\frac{\partial u}{\partial x}+\frac{\partial u}{\partial y}=0$,

(1)

$$
\begin{gathered}
u \frac{\partial u}{\partial x}+v \frac{\partial u}{\partial y}=-k_{0}\left\{u \frac{\partial^{3} u}{\partial x \partial y^{2}}+v \frac{\partial^{3} v}{\partial y^{3}}+\frac{\partial u}{\partial x} \frac{\partial^{2} u}{\partial y^{2}}-\right. \\
\left.\frac{\partial u}{\partial y} \frac{\partial^{2} v}{\partial y^{2}}\right\}+v \frac{\partial^{2} u}{\partial y^{2}}-\frac{\sigma}{\rho} B_{0}{ }^{2} u-\frac{v}{k^{*}} \mathrm{u},
\end{gathered}
$$

$$
\begin{aligned}
& u \frac{\partial T}{\partial x}+v \frac{\partial T}{\partial y}=\frac{k}{\rho C_{p}} \frac{\partial^{2} T}{\partial y^{2}}+\frac{\mu}{\rho C_{p}}\left(\frac{\partial u}{\partial y}\right)^{2}+\frac{\alpha}{\rho C_{p}}\left(u \frac{\partial u}{\partial x} \frac{\partial^{2} u}{\partial y^{2}}+\right. \\
& \left.v \frac{\partial u}{\partial y} \frac{\partial^{2} u}{\partial y^{2}}\right)-\frac{1}{\rho C_{p}} \frac{\partial q_{r}}{\partial y}+\frac{Q_{0}}{\rho C_{p}}\left(T-T_{\infty}\right)+\frac{\sigma}{\rho C_{p}} B_{0}{ }^{2} u^{2}
\end{aligned}
$$

$u \frac{\partial C}{\partial x}+v \frac{\partial C}{\partial y}=D \frac{\partial^{2} C}{\partial y^{2}}-k^{*}\left(C-C_{\infty}\right)$.

With: 
$u(x)=u_{w}(x), v=0, T=T_{w}, C=C_{w} \quad$ at $y=, 0$

(5)

$u(x)=0, u_{y}=0, T=T_{\infty}, C=C_{\infty}$ as $y \rightarrow \infty$

(6)

Where $u$ and $v$ denotes component of velocity, $\mu$ is dynamic viscosity, $\alpha$ is thermal diffusivity, $\mathrm{T}$ is temperature, $\rho$ is density, $\sigma$ is Stefan-Boltzmann constant, $q_{r}$ is radiative heat flux, $B_{0}$ is magnetic field strength, $Q_{0}$ is heat generation/absorption coefficient, $C_{p}$ is the specific heat, $T_{\infty}$ is ambient fluid temperature, $k^{*}$ mean absorption coefficient, $C_{\infty}$ is ambient fluid concentration, $k_{0}$ is short memory coefficient, $D$ is diffusion coefficient and $v$ is Kinematic viscosity.

For radiation via Roseland approximation we have:

$q_{r}=-\frac{4 \sigma}{3 k^{*}} \frac{\partial T^{4}}{\partial y}$

Considering non dimensional forms of momentum, energy and concentration equations, the suitable dimensionless variables introduced are [15]:

$\eta=\sqrt{\frac{u_{w}(x)}{v x}} y, \psi=\sqrt{v x u_{w}(x) f(\eta)}, \theta(\eta)=\frac{T-T_{\infty}}{T_{w}-T_{\infty}}$,

$\emptyset(\eta)=\frac{C-C_{\infty}}{C_{w}-C_{\infty}}$.

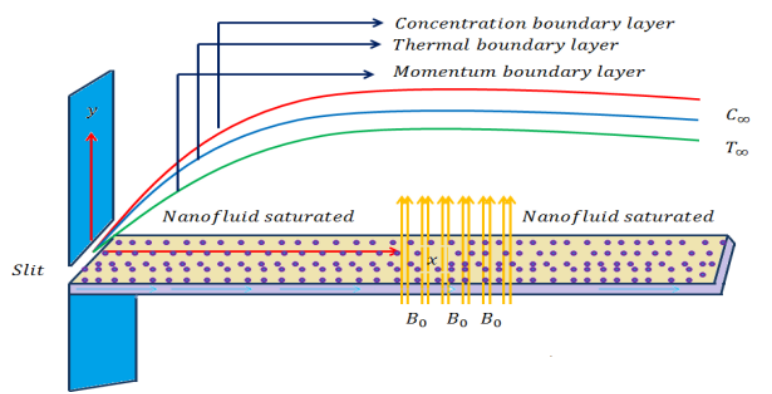

The continuity equation is satisfied identically and the remaining equations take the form:

$f f^{i v}-\left(2 f^{\prime}+\frac{1}{k_{1}}\right) f^{\prime \prime \prime}-\frac{1}{k_{1}}\left(M-k_{2}\right) f^{\prime}-\frac{1}{k_{1}}\left(f^{2}-\right.$

$\left.f f^{\prime \prime}\right)=0$;

$\theta^{\prime \prime}+\operatorname{PrE} c f^{\prime^{2}}+k_{1} \operatorname{PrEc}\left(f^{\prime}{f^{\prime \prime}}^{2}-f f^{\prime \prime} f^{\prime \prime \prime}\right)+R\left[3\left(\theta_{w}-\right.\right.$ 1) $\left.\left\{1+\left(\theta_{w}-1\right) \theta\right\}^{2}\right] \theta^{\prime 2}+\left\{1+\left(\theta_{w}-\right.\right.$

1) $\left.\theta\}^{3} \theta^{\prime \prime}\right]+\delta \operatorname{Pr} \theta+\operatorname{MPrEc}{f^{\prime 2}}^{2}+\operatorname{Pr} \theta^{\prime} f=0$,

(10)

$\emptyset^{\prime \prime}+S c f \emptyset^{\prime}-S c \gamma \emptyset=0$.

With:

$f^{\prime}(0)=1, f(0)=0, f^{\prime}(\infty)=0$ and $f^{\prime \prime}(\infty)=0,(12)$

$\theta(0)=1$ and $\theta(\infty)=0$,

$\emptyset(0)=1$ and $\emptyset(\infty)=0$.

Where $\operatorname{Pr}=\frac{v}{\alpha}$ is Prandtl number, $M=\frac{\sigma B_{0}{ }^{2}}{b \rho}$ is Magnetic field parameter, $k_{1}=\frac{k_{0} b}{v}$ is the Viscoelastic parameter, $k_{2}=\frac{v}{b k^{*}}$ Permeability parameter, $\delta=\frac{Q_{0}}{\rho C_{p} b}$ is Heat generation parameter, $S c=\frac{v}{D}$ is Schmidt number, $E c=$ $\frac{u_{w}{ }^{2}(x)}{C_{p}\left(T_{w}-T_{\infty}\right)}$ is Eckert number $\gamma=\frac{k^{*}}{b}$ chemical reaction parameter and $R e_{x}=\frac{x u_{w}}{v}$ is local Reynold number.

Coefficients of Skin friction $\left(C f_{x}\right)$, Nusselt $\left(N u_{x}\right)$ and Sherwood $\left(S h_{x}\right)$ numbers are:

$C f_{x}=\frac{-2 \tau_{w}}{\rho u^{2} w^{\prime}}$

Fig. 1: Schematic flow diagram. 


$$
\begin{aligned}
& N u_{x}=\left.\left\{-\frac{x}{\left(T_{w}-T_{\infty}\right)} \frac{\partial T}{\partial y}-\frac{16 \sigma^{*} x}{3 k k^{*}\left(T_{w}-T_{\infty}\right)} T^{3} \frac{\partial T}{\partial y}\right\}\right|_{y=0}, \\
& S h_{x}=\left.\left(\frac{x}{\left(C_{w}-C_{\infty}\right)} \frac{\partial C}{\partial y}\right)\right|_{y=0} .
\end{aligned}
$$

Expression of shear stress $\tau_{w}$ at $y=0$ is:

$$
\begin{gathered}
\tau_{w}=\left.\tau_{x y}\right|_{y=0}=\mu b x \sqrt{\frac{b}{v}}\left\{f^{\prime \prime}(0)+\right. \\
\left.\frac{\alpha_{1} b}{\mu}\left(3 f^{\prime}(0) f^{\prime \prime}(0)-f(0) f^{\prime \prime \prime}(0)\right)\right\} .
\end{gathered}
$$

In non-dimensional form Coefficient of skin friction $\left(C f_{x}\right)$, Nusselt $\left(N u_{x}\right)$ and Sherwood $\left(S h_{x}\right)$ numbers are:

$C f_{x} R e_{x}^{0.5}=-2\left\{f^{\prime \prime}(0)+k_{1}\left(3 f^{\prime}(0) f^{\prime \prime}(0)-\right.\right.$ $\left.\left.f^{\prime}(0) f^{\prime \prime}(0)\right)\right\}$,

$$
R e_{x}^{-0.5} N u_{x}=-\left(1+\frac{4}{5} R\left\{1+\left(\theta_{w}-\right.\right.\right.
$$

1) $\left.\theta(0)\}^{3}\right) \theta^{\prime}(0)$,

$R e_{x}^{-0.5} S h_{x}=-\emptyset^{\prime}(0)$.

(21)

\section{SOLUTION PROCEDURE}

Suitable initial approximations are chosen which satisfy the boundary conditions. Following initial guesses, linear operators are taken and homotopic concept is applied to obtain solutions of nonlinear expressions.

$f_{0}(\eta)=1-e^{-\eta}, \theta_{0}(\eta)=e^{-\eta}, \phi_{0}(\eta)=e^{-\eta}$

$£_{f}=f^{\prime \prime \prime}-f^{\prime}, £_{\theta}=\theta^{\prime \prime}-\theta, £_{\phi}=\phi^{\prime \prime}-\phi$.

With:

$£_{f}\left(C_{1}+C_{2} e^{\eta}+C_{3} e^{-\eta}\right)$,

$£_{\theta}\left(C_{4} e^{\eta}+C_{5} e^{-\eta}\right)$
$£_{\phi}\left(C_{6} e^{-\eta}+C_{7} e^{\eta}\right)$.

Where $C_{i},(i=1-7)$ are arbitrary constants, the auxiliary variables $\hbar_{f}, \hbar_{\theta}$ and $\hbar_{\phi}$ have key role in regulating and controlling the convergence region of homotopic expressions. By plotting $\hbar$-curves suitable ranges of these variables are obtained.

\subsection{Convergence analysis}

Homotopy analysis method consists of auxiliary parameters, which control and regulate the region of convergence for homotopic expressions. By plotting $\hbar$-curves (see Fig. 2). Appropriate values of $\hbar_{f}, \hbar_{\theta}$, and $\hbar_{\phi}$ are found in the ranges $-1.6 \leq \hbar_{f} \leq-0.3,-1.4 \leq \hbar_{\theta} \leq-0.4$

and $-1.6 \leq \hbar_{\phi} \leq-0.3$

Table 1 shows convergence numerically.

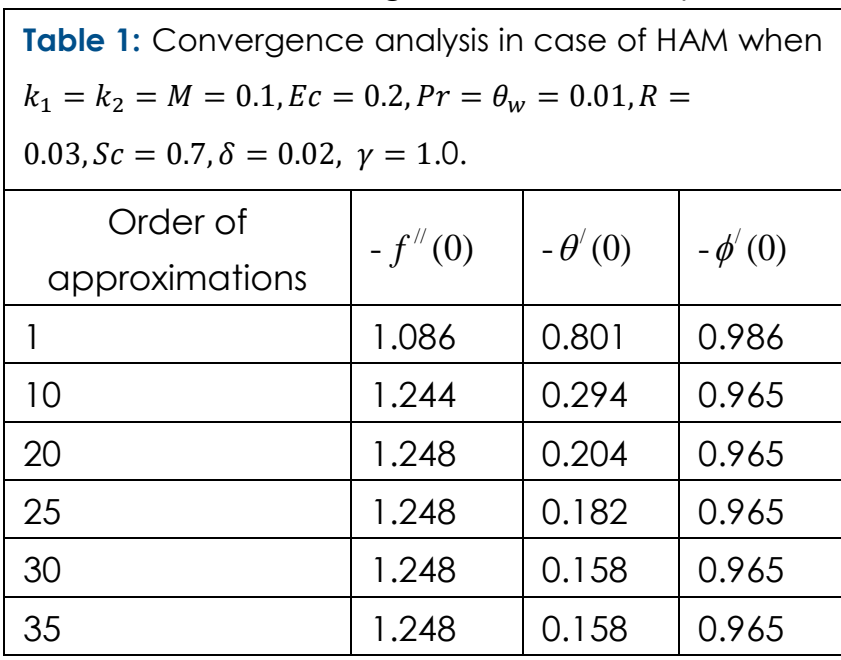

After 15th order of approximations we get convergence of Eq. (9), after 30th order of approximations convergence of Eq. (10) is obtained and 10th order approximation is enough for convergence of Eq. (11). 


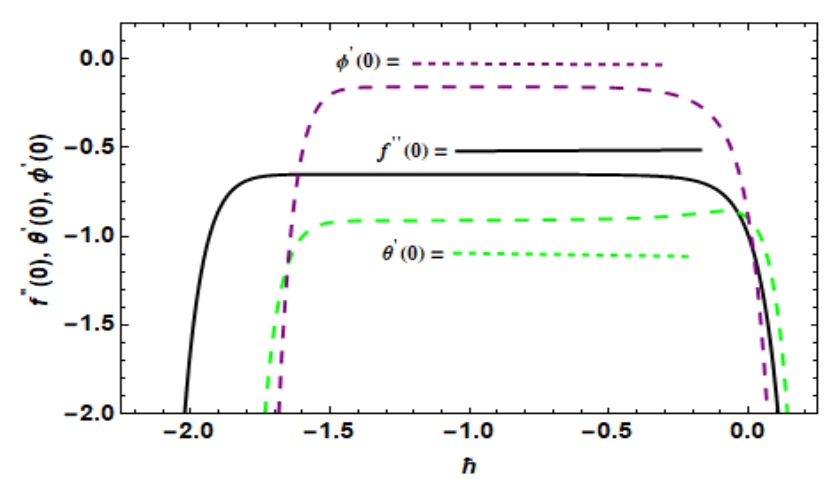

Fig. 2: $\hbar$-curves for velocity, temperature and concentration

\section{RESULTS AND DISCUSSION}

Effect of involved variables versus temperature $(\theta)$, concentration $(\phi)$, skin friction $\left(C f_{x} R e_{x}^{0.5}\right)$, Nusselt and Sherwood numbers $\quad\left(R e_{x}^{-0.5} N u_{x}, R e_{x}^{-0.5} S h_{x}\right) \quad$ are highlighted in this section. The values of involved variables taken for plotting graphs are:

$$
k_{1}=k_{2}=M=0.1, E c=0.2, \operatorname{Pr}=\theta_{w}=0.01, R=
$$

$0.03, \delta=0.02, S c=0.7$ and $\gamma=1$.

Fig. 3 explains the curves of $f^{\prime}$ for $k_{1}$. Here for greater values of $k_{1}, f^{\prime}$ and its related layer is thicker. Velocity $f^{\prime}$ increases when $k_{1}$ is increased. In fact liquid viscosity diminishes via an increment in $k_{1}$ which yields higher $f^{\prime}$. Fig. 4 shows the variation in $f^{\prime}$ against $k_{2}$. The thermal layer and $f^{\prime}$ increase with the increase in $k_{2}$. Ultimately there is an increment in $f^{\prime}$ for larger values of $k_{2}$. Fig. 5 represents the effect of $M$ on $f^{\prime}$. Larger values of $M$ improves both velocity and related layer thickness. Here we observe an improvement in $f^{\prime}$. Fig. 6 shows that $\theta$ and thickness of related layer diminish for greater values of $k_{1}$. The effect of $\operatorname{Pr}$ number on temperature distribution is depicted in Fig. 7. The related layer and temperature distribution decreases as $\mathrm{Pr}$ increases. Importance of Sc is shown in Fig. 8. Here larger Sc increases the concentration and thickness of related layer. Fig 9 witnesses that $\phi$ and thickness of corresponding layer decreases with the increase of $\gamma$.

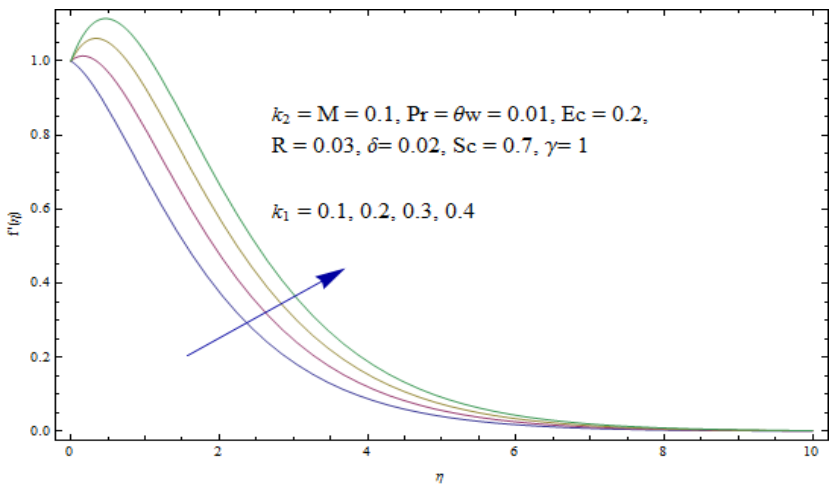

Fig. 3: Influence of $k_{1}$ on velocity distribution.

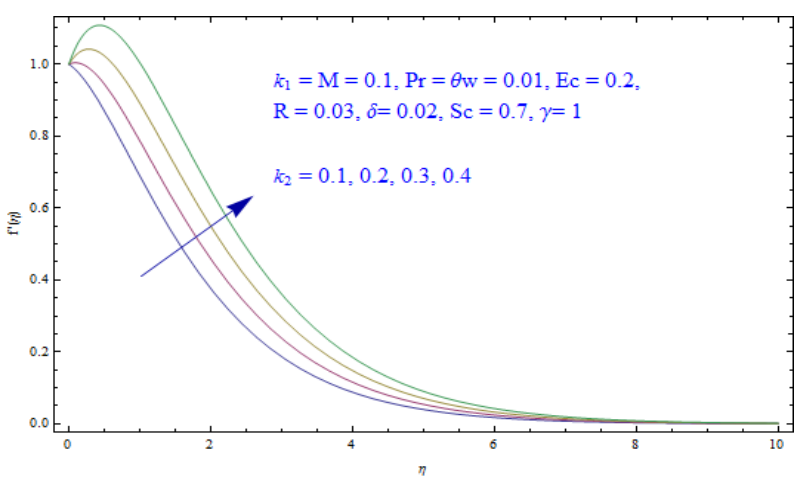

Fig. 4: Influence of $k_{2}$ on velocity distribution. 


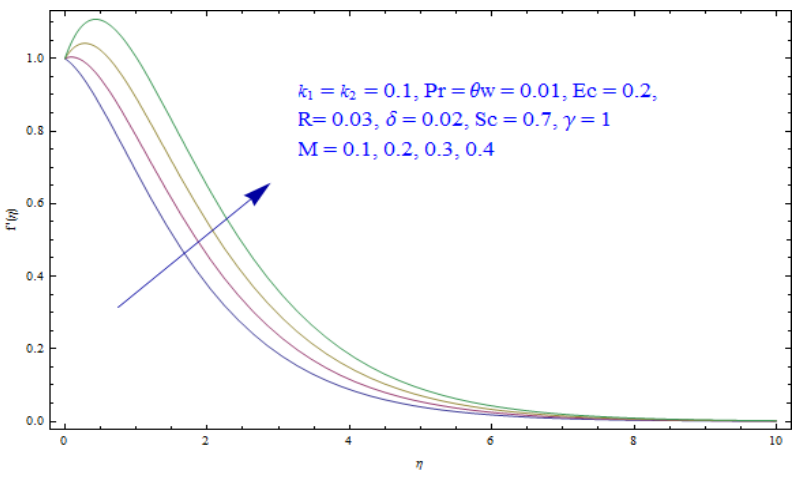

Fig. 5: Influence of $M$ on velocity distribution.

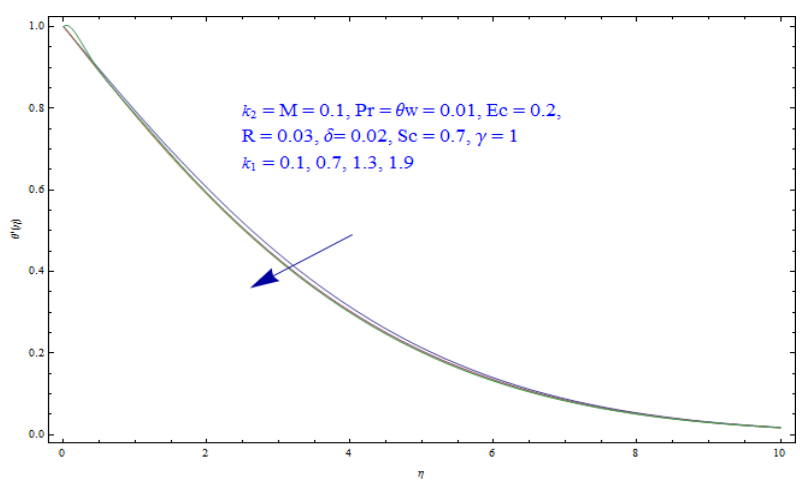

Fig. 6: Influence of $k_{2}$ on temperature distribution.

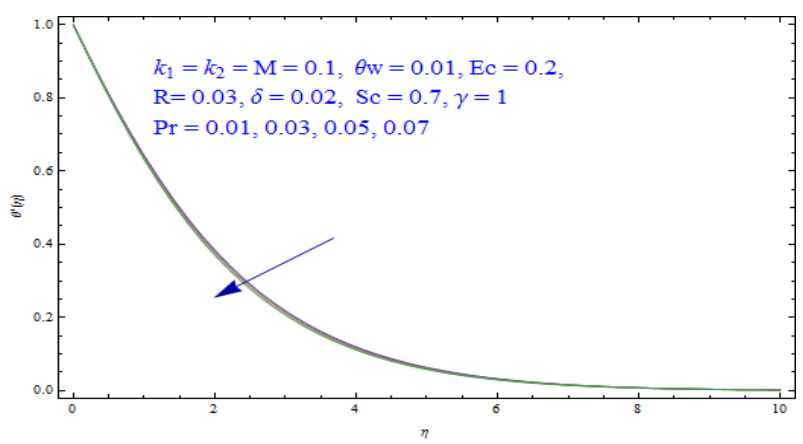

Fig. 7: Influence of Pr on temperature distribution.

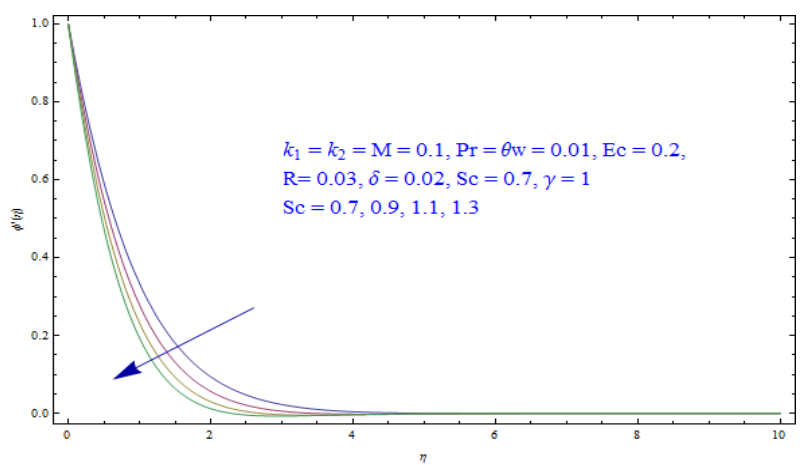

Fig. 8: Influence of Sc on concentration distribution.

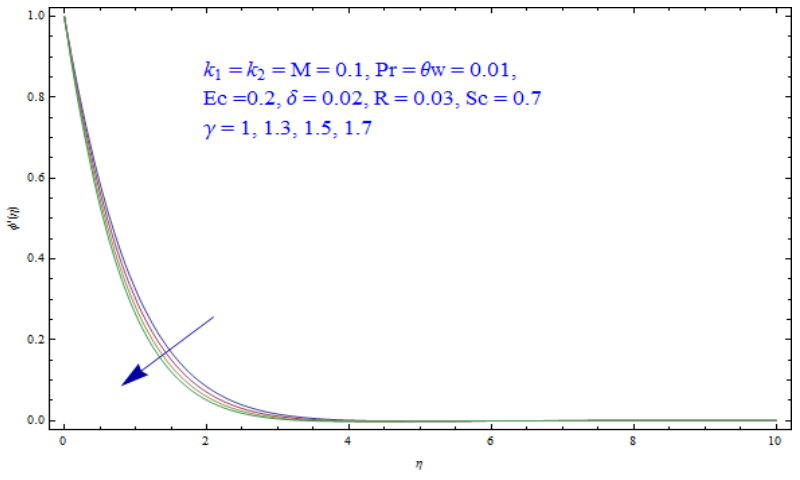

Fig. 9: Influence of $\gamma$ on concentration distribution.

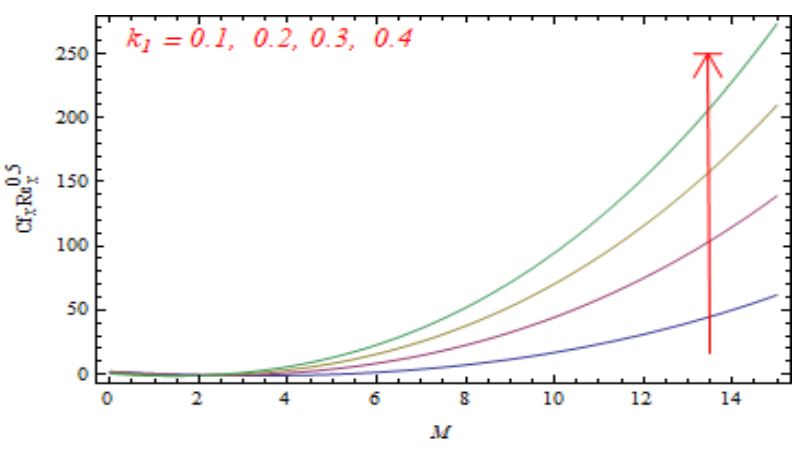

Fig. 10: Effects of $k_{1}$ and $M$ on $R e_{x}{ }^{0.5} C f_{x}$.

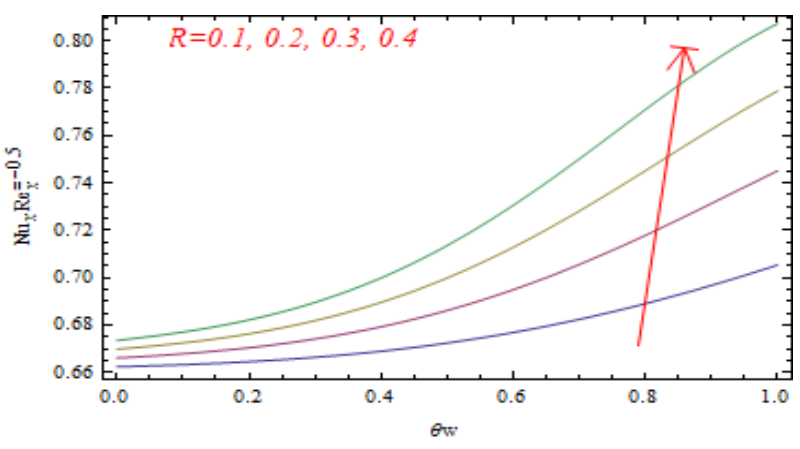

Fig. 11: Effects of $\mathrm{R}$ and $\theta_{w}$ on $R e_{x}{ }^{-0.5} N u_{x}$.

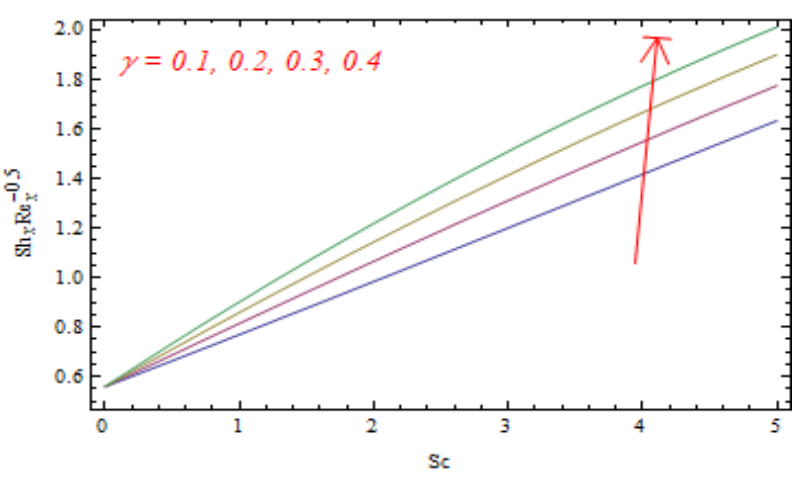

Fig. 12: Effects of SC and $\gamma$ on $R e_{x}{ }^{-0.5} S h_{x}$. 
Fig. 10 Show that larger $k_{1}$ and $M$ corresponds to higher $R e_{x}{ }^{0.5} C f_{x}$. and associated layer. Fig. 11 Show that for larger $\theta \omega$ and $\mathrm{R}$ corresponds to higher $R e_{x}{ }^{-0.5} N u_{x}$. Fig. 12 divulges the variation of $\gamma$ and Sc against $S h_{x} \operatorname{Re}_{x}^{-0.5}$. Higher values of $\gamma$ and Sc reported higher $R e_{x}{ }^{-0.5} S h_{x}$.

\section{CONCLUSIONS}

Here we study nonlinear radiation and mixed convection aspects in MHD boundary layer visco-elastic $2^{\text {nd }}$ grade fluid flow over a moving stretching sheet in a pours medium. The formulated equations are transformed to ordinary differential equations by taking suitable transformations. The solution of governing equations is obtained by using HAM.

It is observed that visco-elastic parameter effects velocity and temperature distribution, the velocity increases and temperature decreases in boundary-layer. This shows the effect of visco-elastic parameter. The magnetic field parameter increases velocity distribution it has no any effect on temperature and concentration. The prandtl number has no effect on velocity and concentration but it decreases the temperature distribution. The permeability parameter increases the velocity distribution. The Renold number, Ecrect number and $\theta_{w}$ have no any effect on velocity, temperature and concentration distributions. The Schmidt number and radiation parameter $\gamma$ decreases concentration distribution.

\section{REFERENCES}

[1] Alfvén, H., Existence of Electromagnetic-Hydrodynamic Waves. Nature, (1942). 150: p. 405-406.

[2] Kumari, M., H.S. Takhar, and G. Nath, MHD flow and heat transfer over a stretching surface with prescribed wall temperature or heat flux. Wärme und Stoffübertragung, (1990). 25(6): p. 331-336.

[3] Andersson, H.I., MHD flow of a viscoelastic fluid past a stretching surface. Acta Mechanica, (1992). 95(1): p.227-230.

[4] Bhatti, M.M., et al., Entropy Generation on MHD Eyring-Powell Nanofluid through a Permeable Stretching Surface. Entropy, (2016). 18(6).

[5] Attia, H.A., Unsteady MHD flow near a rotating porous disk with uniform suction or injection. Fluid Dynamics Research, (1998). 23(5): p. 283-290.

[6] Afify, A.A., MHD free convective flow and mass transfer over a stretching sheet with chemical reaction. Heat and Mass Transfer, (2004). 40(6): p. 495-500.

[7] Vajravelu, K., K.V. Prasad, and S.R. Santhi, Axisymmetric magnetohydrodynamic (MHD) flow and heat transfer at a non-isothermal stretching cylinder. Applied Mathematics and Computation, (2012). 219(8): p. 3993-4005.

[8] Received: $03 \quad$ November 2016. Revised/Accepted: 29 December (2016).

[9] Nadeem, S., R.U. Haq, and C. Lee, MHD boundary layer flow over an unsteady shrinking sheet: analytical and numerical approach. 
Journal of the Brazilian Society of Mechanical Sciences and Engineering, (2015). 37(4): p. 1339-1346.

[10]. Sheikholeslami, M., et al., Heat transfer and turbulent simulation of nanomaterial due to compound turbulator including irreversibility analysis. International Journal of Heat and Mass Transfer, 2019. 137: p. (1290-1300).

[11] Abel, S., K.V. Prasad, and A. Mahaboob, Buoyancy force and thermal radiation effects in MHD boundary layer visco-elastic fluid flow over continuously moving stretching surface. International Journal of Thermal Sciences, (2005). 44(5): p. 465-476.

[12].Ghosh, N.C., B.C. Ghosh, and L. Debnath, The hydromagnetic flow of a dusty visco-elastic fluid between two infinite parallel plates. Computers \& Mathematics with Applications, (2000), 39: p. 103-116.

[13] Datti, P.S., et al., MHD visco-elastic fluid flow over a non-isothermal stretching sheet. International Journal of Engineering Science, (2004), 42(8): p. 935-946.

[14] Bég, O.A., et al., Free convection heat and mass transfer from an isothermal sphere to a micropolar regime with Soret/Dufour effects. International Journal of Heat and Mass Transfer, (2011), 54(1): p. 9-18.

[15] Kamel, M.H., Unsteady MHD convection through porous medium with combined heat and mass transfer with heat source/sink. Energy Conversion and Management, (2001), 42(4): p. 393-405.

[16] Chen, C.-H., Effects of magnetic field and suction/injection on convection heat transfer of non-Newtonian power-law fluids past a power-law stretched sheet with surface heat flux. International Journal of Thermal Sciences, (2008), 47(7): p. 954-961.

[17] Malik, M.Y., et al., Magnetohydrodynamic flow of Sisko fluid over a stretching cylinder with variable thermal conductivity: A numerical study. AlP Advances, (2016), 6(2): p. 025316.

[18] Liao, S., On the homotopy analysis method for nonlinear problems. Applied Mathematics and Computation, (2004), 147(2): p. 499-513.

[19] Khan, M. and A. Shahzad, On axisymmetric flow of Sisko fluid over a radially stretching sheet. International Journal of Non-Linear Mechanics, (2012), 47(9): p. 999-1007.

[20] Hayat, T., et al., Joule heating and viscous dissipation in flow of nanomaterial by a rotating disk. International Communications in Heat and Mass Transfer, (2017), 89: p. 190-197.

[21] Rashidi, M.M., E. Momoniat, and B. Rostami, Analytic Approximate Solutions for MHD Boundary-Layer Viscoelastic Fluid Flow over Continuously Moving Stretching Surface by Homotopy Analysis Method with Two Auxiliary Parameters. Journal of Applied Mathematics, (2012), 2012: p. 780415.

[22]. Abbas, Z., et al., Mixed convection in the stagnation-point flow of a Maxwell fluid towards a vertical stretching surface. Nonlinear Analysis: Real World Applications, 2010. 11 (4): p. 3218-3228. (2003) 3,154,026, May 15.

Received: 13 February 2019. Revised/Accepted: 3 April 2019. 